\title{
Magneto-optical spectra of long range chemically ordered FePd(001) alloy films
}

\author{
G. Armelles, ${ }^{\text {a) }}$ D. Weller, and B. Rellinghaus \\ IBM Research Division, Almaden Research Center, 650 Harry Road, San Jose, \\ California 95120-6099 \\ P. Caro, A. Cebollada, and F. Briones \\ Centro Nacional de Microelectrónica-IMM (CSIC), Isaac Newton 8 (PTM), Tres Cantos, \\ Madrid 28760, Spain
}

(Received 5 February 1997; accepted for publication 1 July 1997)

\begin{abstract}
Magneto-optical and magnetic properties of sputtered epitaxial FePd (001) alloy films have been studied as a function of the film deposition temperature. Characteristic changes in the magnetic anisotropies and Kerr effect spectra are observed and correlated with the degree of long range chemical ordering. (C) 1997 American Institute of Physics. [S0021-8979(97)05819-2]
\end{abstract}

\section{INTRODUCTION}

The occurrence of chemical ordering can have a dramatic influence on structural, electronic, and magnetic properties in many materials systems. Examples of recent interest are binary metallic alloy systems such as $\mathrm{CoPt},{ }^{1-4} \mathrm{FePt}^{2-7}$ and $\mathrm{FeAu}$, 8 where strong interdependencies between magnetic and magneto-optical properties and the degree of long range chemical ordering have been observed. Near the equiatomic composition, these ordered alloys form a $\mathrm{CuAu}$ $\left(L 1_{0}\right)$ crystal structure, i.e., a face centered lattice with pure $\mathrm{Cu}$ and $\mathrm{Au}$ planes stacked along the [001] direction. Such natural superlattice phases exhibit strong tetragonal distortions along the [001] axis often accompanied by large magnetic anisotropies and enhanced magneto-optical effects, as it was shown most evidently for FePt. ${ }^{2-7}$ It was further shown, that these ordered metallic alloys can be obtained employing thin film growth techniques ${ }^{1-9}$ at temperatures well below the bulk disorder-order transition temperature. This together with relatively large magnetic anisotropies make them potential candidates for future ultrahigh density magnetic recording disk applications. ${ }^{10}$

In this article, we discuss magnetic and magneto-optical properties of FePd. This is another but less explored example of an ordered phase with the $\mathrm{CuAu}\left(L 1_{0}\right)$ crystal structure. ${ }^{11}$ Strong magnetic anisotropies are known in bulk FePd alloys ${ }^{12-15}$ and electronic property changes as function of chemical order, as e.g., reflected in magneto-optical Kerr effect (MOKE) spectra, may well be expected. Polar MOKE spectra of disordered FePd alloys have already been measured by Buschow et al. ${ }^{16}$ and by Reim et al. ${ }^{17}$ Characteristic features in the infrared (1.1-1.5 eV) and ultraviolet (4-4.5 eV) spectral ranges were found and tentatively attributed to transitions between Fe-like and Pd-like states, ${ }^{17}$ respectively. Visokay et al. ${ }^{18}$ have performed a detailed transmission electron microscopy study in ordered samples obtained by annealing Fe/Pd multilayers. They also present a Kerr rotation spectrum of an ordered sample in the spectral range of the Fe-like transitions. Recently, Sürgers et al. ${ }^{19}$ reported a small

\footnotetext{
${ }^{a)}$ Present address: CNM-IMM (CSIC) Isaac Newton 8 (PTM), Tres Cantos, Madrid 28760, Spain. Electronic mail: gaspar@imm.cnm.csic.es
}

increase of the Kerr rotation for the Fe-like peak in samples with partial chemical ordering but did not address the Pd-like transitions. The present article discusses MOKE spectra in both the infrared and ultraviolet ranges and reports a quantitative analysis of the degree of long range chemical ordering and magnetic anisotropy in sputtered epitaxial FePd films.

\section{EXPERIMENT}

The samples were deposited in a ultrahigh vacuum (UHV) triode-sputtering system at constant Ar pressure of $4 \times 10^{-4}$ mbar during film growth. The deposition rates were about $0.2 \AA /$ s. As substrates we used mechanically polished $\mathrm{MgO}$ (001) single crystals (Marketech International), with a maximum misorientation of $0.5^{\circ}$ and a rms roughness of $4 \AA$. Prior to the FePd growth, a $300 \AA$ buffer layer of Pt was deposited at $700{ }^{\circ} \mathrm{C}$. At such thickness, a continuous Pt seed layer is formed. ${ }^{20}$ In situ reflection of high energy electron diffraction RHEED measurements confirmed that Pt adopts a (100) orientation. The substrate temperature was then reduced to different values between room temperature (RT) and $500{ }^{\circ} \mathrm{C}$ and a $1000-\AA ̊ \AA$-thick FePd alloy film was grown by co-sputtering from independent $\mathrm{Fe}$ and Pd targets. The deposition was completed with a Pt cap layer of 30-50 thickness, grown at RT and intended to prevent oxidation.

The films were characterized ex situ by x-ray diffraction (XRD) taken with $\mathrm{Cu} K_{\alpha 1}$ radiation. Compositions and thicknesses were checked with electron microprobe and Rutherford backscattering spectrometry (RBS) measurements. Magnetization and magnetic anisotropy measurements were carried out by standard vibrating sample (VSM) and torque magnetometries. MOKE spectra (polar Kerr angle and ellipticity) were measured at room temperature in the $0.8-5.4 \mathrm{eV}$ photon energy range using a fully automated spectrometer described elsewhere. ${ }^{21}$

\section{RESULTS}

As expected, the degree of chemical ordering is found to depend strongly on the deposition temperature. This is exemplified in Fig. 1(a) where we show symmetric XRD scans for 
(a)

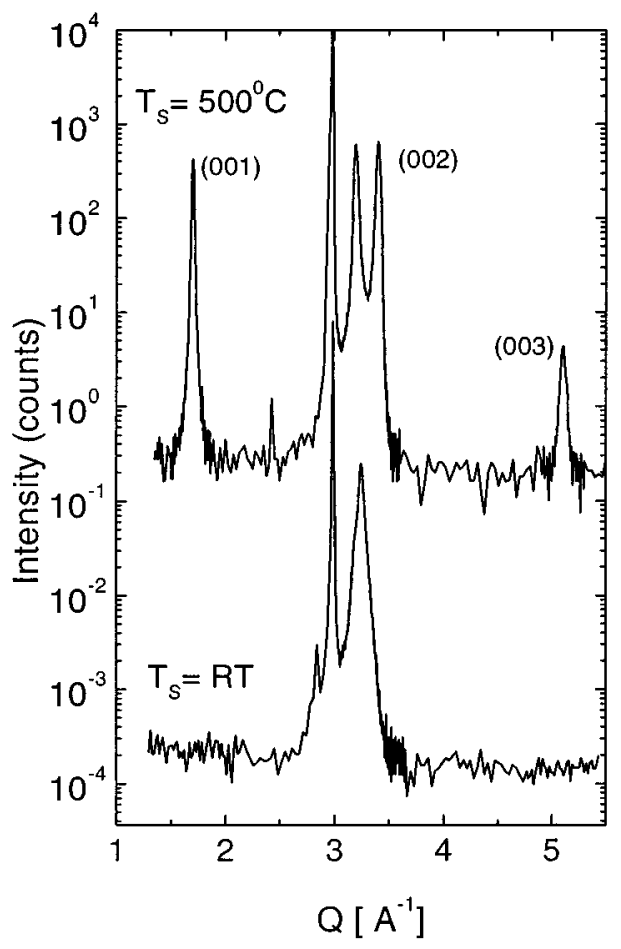

POLAR
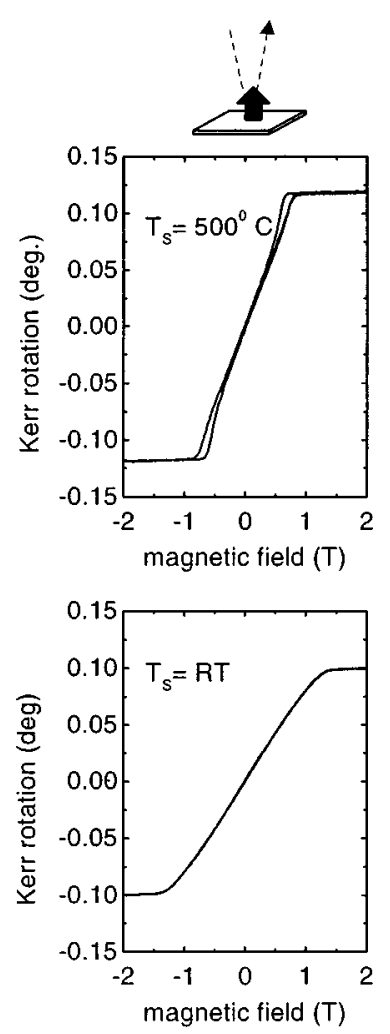

TRANSVERSE
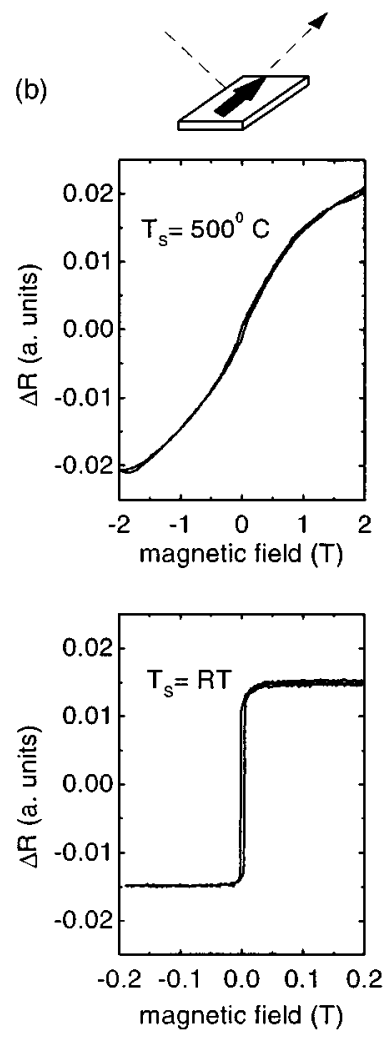

FIG. 1. (a) Symmetric XRD scans of two FePd (001) samples grown at $500{ }^{\circ} \mathrm{C}$ and RT. (b) Polar and transverse Kerr loops from the corresponding films.

the two extreme cases of a chemically disordered (bottom scan) and an almost completely chemically ordered FePd film (top scan), respectively.

In the sample grown at $500{ }^{\circ} \mathrm{C}$ the fundamental (002) peak is accompanied by (001) and (003) superlattice reflections originating from the presence of the ordered phase. Additionally a substrate peak at $2.98 \AA^{-1}$ and a Pt peak at $3.20 \AA^{-1}$ are observed. The intensity of the (001) and (003) superstructure peaks indicate a high degree of chemical ordering with the tetragonal $c$ axis aligned along the film normal. The positions of these peaks gives an average out of plane spacing of $c=3.694 \AA$. Narrow rocking curve widths full width half maximum (FWHM) of only $0.79^{\circ}$ from these peaks demonstrate a high degree of crystallographic orientation. The long-range chemical ordering parameter, $S,{ }^{6,22}$ was quantified using integrated intensities from the (001), (002), and (003) peaks extracted from fits to the experimental data. This procedure is described in detail elsewhere. ${ }^{23} S$ is equal to 1 for perfectly ordered alloys of the equiatomic composition and vanishes for completely chemically disordered alloys. In the sample grown at $500{ }^{\circ} \mathrm{C}, S$ was found to be 0.8 . This value decreases as the growth temperature is reduced. The sample grown at RT shows besides the $\mathrm{MgO}(200)$ substrate peak at $2.98 \AA^{-1}$ one additional peak with a shoulder. The shoulder corresponds to the (200) reflection of the Pt buffer layer. A deconvolution of that shoulder with the main peak gives an out-of-plane lattice parameter for the RT film of $3.87 \AA \pm 2 \%$. The absence of (001) or (003) reflections indicates chemical disorder.
Figure 1(b) shows polar (out-of-plane) and transverse (in-plane) Kerr hysteresis loop measurements of the above samples. The measurements were performed with a $633 \mathrm{~nm}$ $\mathrm{HeNe}$ laser system at RT in normal and $45^{\circ}$ incidence angle geometry, respectively. In the polar case one measures the Kerr rotation angle while the transverse Kerr effect detects a change in reflectance. Both measurements are in principle proportional to the sample magnetization. We observe significant differences in the overall magnetic behavior between the two samples. The disordered film has an in-plane easy axis, whereas the ordered sample appears to have an easy magnetization axis perpendicular to the plane of the film. Clearly, the saturation field in the polar configuration is lower in the ordered case. Conversely, we were not able to saturate the ordered film with a field up to $2 \mathrm{~T}$ aligned within the film plane, while the disordered sample shows soft inplane easy axis behavior. These observations are corroborated by quantitative torque and vibrating sample magnetometry measurements. From a Fourier analysis of the torque curves, measured in a $2 \mathrm{~T}$ field rotating through the sample normal, we deduce out-of-plane anisotropy constants of $K_{1}$ $=9.3310^{6} \mathrm{erg} / \mathrm{cc}$ and $K_{2}=2.8610^{6} \mathrm{erg} / \mathrm{cc}$ for the ordered FePd(001) film. Here, the intrinsic first order anisotropy constant was determined in the usual way by adding the stray field term $2 \pi M_{s}^{2}$ to the measured effective anisotropy $K_{1}^{\text {eff }}: K_{1}=K_{1}^{\text {eff }}+2 \pi M_{s}^{2}$. $M_{s}$ denotes the saturation magnetization, which was measured to be $M_{s}=1100 \mathrm{emu} / \mathrm{cc}$. The values of $M_{s}$ are the same for the ordered and disordered films. 


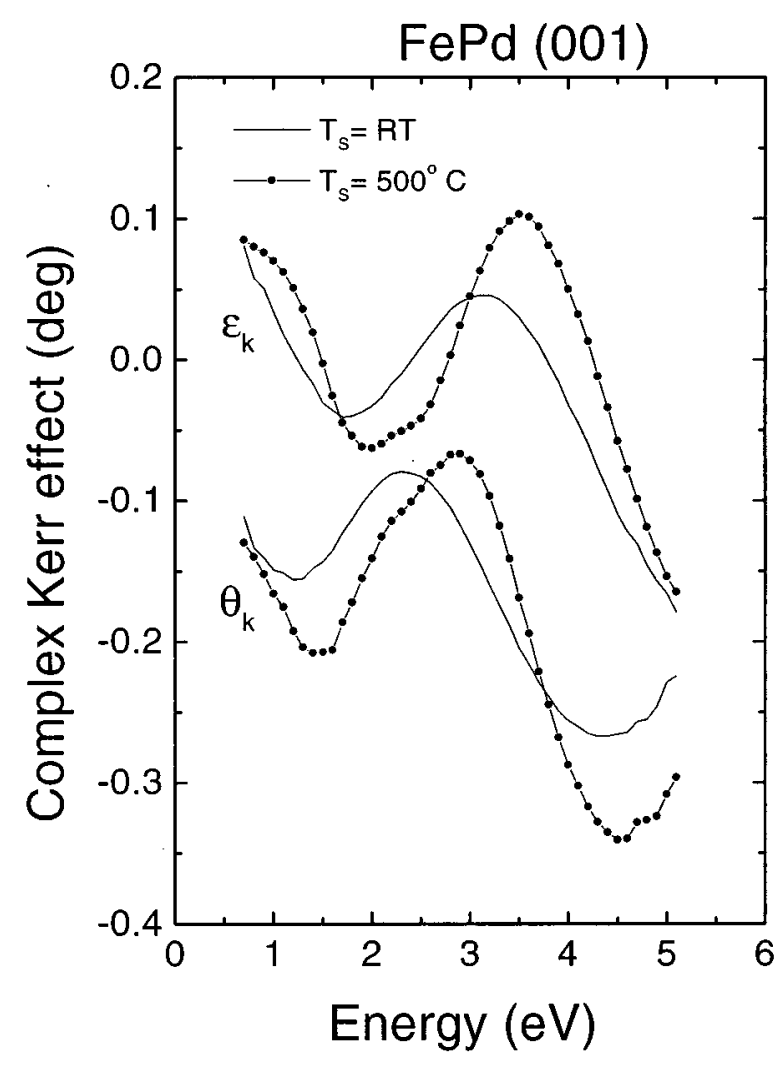

FIG. 2. Complex polar magneto-optical Kerr spectra of the two samples described in Fig. 1.

There is also a $90^{\circ}$ phase shift of the twofold component of the torque curves of the disordered film with respect to the ordered film, which confirms the change of the easy axis from in-plane (disordered film) to a perpendicular easy axis (ordered film). The anisotropy constants of the ordered film $(S-0.8)$ are actually smaller than those reported for bulk material $\left[(1-3) \times 10^{7} \mathrm{erg} / \mathrm{cc}\right]$ which corresponds to completely ordered samples $(S=1)$. No significant in-plane anisotropy was found.

The growth temperature has obviously a paramount effect on the structural and magnetic properties in this system. The observation of an out-of-plane easy axis is clearly attributable to the evolution of chemical ordering, with the tetragonal $c$ axis of the structure perpendicular to the film plane.

Figure 2 shows polar MOKE spectra (Kerr angle and ellipticity) for the present FePd samples. The Kerr spectra were taken in the presence of an external field of $\pm 2 \mathrm{~T}$, sufficient to saturate both samples in the film normal direction. The spectral features of the ordered sample are sharper than the equivalent features of the disordered film. In particular, as the degree of chemical ordering increases, the features in the Kerr rotation spectra located at 1.1 and $4.2 \mathrm{eV}$ shift towards higher transition energies, and the intensity increases. This effect has already been found in $\operatorname{FePt}(001)$ alloys $^{7}$ and again concurs with the presence of chemical ordering and perpendicular magnetic anisotropy. The relative intensity changes in FePd are found to be similar to the ones previously reported in FePt in the UV (4-4.5 eV) region (Pd-like transitions and Pt-like transitions, respectively), but

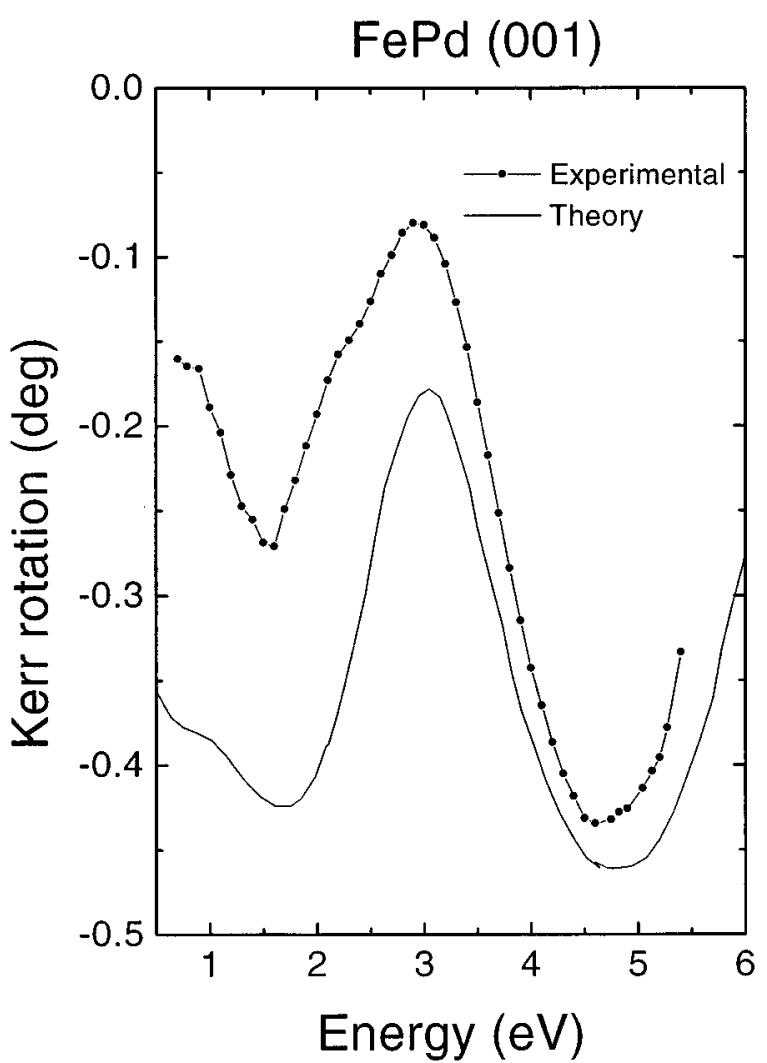

FIG. 3. Comparison of theoretical Kerr rotation spectrum calculated using $a b$ initio theory (Ref. 25) and experimental Kerr rotation spectrum of the $80 \%$ chemically ordered sample grown at $500{ }^{\circ} \mathrm{C}$.

are much less pronounced in the IR region $(1-1.5 \mathrm{eV})$, where one expects the spectra to be dominated by Fe-like transitions. ${ }^{17,24}$ This different behavior between FePd and FePt suggest a much higher degree of $\mathrm{Fe}-\mathrm{Pt}$ than $\mathrm{Fe}-\mathrm{Pd}$ hybridization.

Finally, in Fig. 3, we compare the theoretical Kerr rotation spectrum obtained from $a b$ initio MOKE calculations for a completely ordered FePd alloy ${ }^{25}$ with our experimental results (ordering parameter $S=0.8$ ). The calculated Kerr spectrum reproduces the positions of the experimentally observed two spectral features, however, their relative intensities are different. Note that in that figure the experimental Kerr rotation spectrum has been corrected for the presence of a $30-\AA$-thick Pt cap layer. In the region of the Pd-like transitions (UV region) the agreement between theory and experiment is almost quantitative. This is in contrast with previous theory-experiment comparisons using data of disordered samples ${ }^{25}$ where neither the intensity nor the energy position of the Pd-like feature were reproduced. In the IR region, the comparison is much less favorable. Although the energy positions agree, the experimental Kerr rotation is greatly reduced. The origin of this discrepancy is unclear. Part of it could be attributed to free carriers contributing to the experimental spectrum (intraband transitions) and neglected in the present $a b$ initio calculations. ${ }^{25}$ Further of course, the present sample was only about $80 \%$ ordered, while the calculation assumes full chemical ordering. 


\section{CONCLUSION}

In summary, ordered and disordered $\mathrm{FePd}(001)$ alloys are obtained by co-sputtering of $\mathrm{Fe}$ and $\mathrm{Pd}$ at different substrate temperatures. Deposition temperatures of $500{ }^{\circ} \mathrm{C}$ result in highly chemically ordered samples with an order parameter up to 0.8 in the present case. Characteristic changes in the polar MOKE spectra, both in the infrared and ultraviolet ranges, correlate with the degree of chemical ordering. The ordered samples have a perpendicular magnetic easy axis with large out-of-plane anisotropy constants.

\section{ACKNOWLEDGMENTS}

G.A. thanks the DGICYES (Spain) for financial support. The authors would like to thank M. Toney for his help in the $\mathrm{x}$-ray characterization.

${ }^{1}$ G. R. Harp, D. Weller, T. A. Rabedeau, R. F. C. Farrow, and R. F. Marks, Mater. Res. Soc. Symp. Proc. 313, 493 (1993).

${ }^{2}$ B. M. Lairson and B. M. Clemens, Appl. Phys. Lett. 63, 1438 (1993).

${ }^{3}$ B. M. Lairson, M. R. Visokay, S. Sinclair, and B. M. Clemens, Mater. Res. Soc. Symp. Proc. 313, 805 (1993).

${ }^{4}$ M. R. Visokay and R. Sinclair, Appl. Phys. Lett. 66, 1692 (1995).

${ }^{5}$ B. M. Lairson, M. R. Visokay, R. Sinclair, and B. M. Clemens, Appl. Phys. Lett. 62, 639 (1993).

${ }^{6}$ Cebollada, D. Weller, J. Sticht, G. R. Harp, R. F. C. Farrow, R. F. Marks, R. Savoy, and J. C. Scott, Phys. Rev. B 50, 3419 (1994).

${ }^{7}$ S. Mitani, K. Takanashi, M. Sano, H. Fujimori, A. Osawa, and H. Nakajima, J. Magn. Magn. Mater. 148, 163 (1995).
${ }^{8}$ K. Takanashi, S. Mitani, M. Sano, H. Fujimori, H. Nakajima, and A. Osawa, Appl. Phys. Lett. 67, 1016 (1995).

${ }^{9}$ S. Mitani, K. Takanashi, H. Nakajima, K. Sato, R. Schreiber, P. Grünbreg, and H. Fujimori, J. Magn. Magn. Mater. 156, 7 (1996).

${ }^{10}$ K. R. Coffey, M. A. Parker, and J. K. Howard, IEEE Trans. Magn. 31, 2737 (1995)

${ }^{11}$ Hansen, Constitution of Binary Alloys (McGraw-Hill, New York, 1958).

${ }^{12}$ L. M. Magat, A. S. Yermolenko, G. V. Ivanova, G. M. Makarova, and YA. S. Shur, Fiz. Met. Metalloved. 26, 132 (1968).

${ }^{13}$ V. V. Maykov, A. Ye. Yermanov, G. V. Ivanov, V. I. Khrabrov, and L. M. Magat, Fiz. Met. Metalloved. 67, 79 (1989); 67, 76 (1989).

${ }^{14}$ A. Ye. Yermanov and V. V. Maykov, Fiz. Met. Metalloved. 5, 201 (1990); 69, 198 (1990).

${ }^{15}$ T. Klemmer, D. Hoydick, H. Okumura, B. Zhang, and W. A. Soffa, Scr. Metall. Mater. 33, 1793 (1995).

${ }^{16}$ K. H. J. Buschow, P. G. van Engen, and R. Jongebreur, J. Magn. Magn. Mater. 38, 1 (1983)

${ }^{17}$ Reim, H. Brandle, D. Weller, and J. Schoenes, J. Magn. Magn. Mater. 93, 220 (1991).

${ }^{18}$ M. R. Visokay, B. M. Lairson, B. M. Clemens, and R. Sinclair, J. Magn. Soc. Jpn. 19, 399 (1995).

${ }^{19}$ C. Sürgers, E. Kay, and S. X. Wang, J. Appl. Phys. 80, 5753 (1996).

${ }^{20}$ P. Caro, A. Cebollada, D. Ravelosona, J. Tamayo, R. Garcia, F. Briones, Acta Materialia (submitted).

${ }^{21}$ D. Weller, Spin-Orbit-Influenced Spectroscopies of Magnetic Solids, Lecture Notes in Physics 466 (Springer, Berlin, 1996).

${ }^{22}$ B. E. Warren, X-ray Diffraction (Dover, New York, 1990), p. 208.

${ }^{23}$ Farrow, D. Weller, R. F. Marks, M. F. Toney, A. Cebollada, and G. R. Harp, J. Appl. Phys. 79, 5967 (1996).

${ }^{24}$ T. Katayama, T. Sugimoto, Y. Suzuki, M. Hashimoto, P. de Hann, and J. C. Lodder, J. Magn. Magn. Mater. 104-107, 1002 (1992).

${ }^{25}$ I. Osterloh, P. M. Oppeneer, J. Sticht, and J. Kubler, J. Phys.: Condens. Matter 6, 285 (1994). 